\title{
Multi Frequency Approach to Analog Fault Diagnosis using Pole Sensitivity Analysis
}

\author{
S.P. Venu Madhava Rao ${ }^{1}$ O. Manasa ${ }^{2}$ \\ ${ }^{1}$ Professor and Head, Department of ECE, SNIST, Hyderabad \\ spvenu@yahoo.com \\ ${ }^{2}$ Student, M.Tech (VLSI and ES), SNIST, Hyderabad \\ kytee.manasa@gmail.com
}

\begin{abstract}
In this paper an efficient algorithm using multi-frequency approach has been applied to fault diagnosis of analog electronic circuits using Pole Sensitivity analysis. Here after the application of the multi frequency approach to the pole sensitivity analysis the results show that the fault diagnosis of the circuit has increased. This has been proved by using a Band Pass Filter.
\end{abstract}

Keywords: Pole Sensitivity, Zero Sensitivity, Multi frequency, Fault Diagnosis, Ambiguity table.

\section{Council for Innovative Research}

Peer Review Research Publishing System

Journal: International Journal of Management \& Information Technology

Vol. 7, No. 1

editor@cirworld.com

www.cirworld.com, member.cirworld.com

\section{INTRODUCTION}


The increasing complexity of the System-on-chip (SOC) makes it very important for the accuracy and consistency in timing analysis and clock distribution. The importance of the analog circuits is increasing, the main reason is that the digital systems need analog circuits to interface to the real world which is analog in nature. Analog circuits have in fact expanded with the digital circuits into new applications. Analog circuits occupy only a small fraction of the total area of an integrated circuit. But the performance of analog circuits is very critical in the performance of the system. Analog integrated circuit fault diagnosis is thus very important.

Analog Fault Diagnosis has been of immense research interest for the past three decades and continues to sustain the same zeal even today. The main challenges today in analog fault diagnosis are to design universally accepted fault models, cost effective, faster and accurate diagnosis of faults. Importantly all this is desired even in the presence of inherent characteristics of analog circuits like tolerances, non-linearity and in- accessible test nodes etc.

S.P. Venu Madhava Rao, N.S.C. Babu and K.Lal Kishore [1] have proposed a novel node-frequency approach in analog fault diagnosis which resulted in more faults being diagnosed. In [2] a circuit has been designed and developed using reconfigurable architecture to diagnose faults in analog circuits. N.S.C. Babu, S.P. Venu Madhava Rao and others in [3] proposed an efficient algorithm that improves the existing multi-frequency approach in diagnosing more faults at a faster rate.

T.N. Trick and Y.Li proposed a new first order sensitivity fault isolation algorithm [4]. The independence of the sensitivity vectors indicates that the test points are capable of identifying faults. The degree of the testability of the circuit under test is determined by the rank of the sensitivity matrix of the columns.

L.Rapisarda and R. DeCarlo [5] used component connection mode in the development of multi frequency problem. This method made the solution process simple, as the equations now have a fixed polynomial order which is generally quadratic. The developed method also includes a test for diagnosability. John W. Bandler and others [6] made an exhaustive and comprehensive review of the fault location techniques in analog electronic circuits. The four main approaches discussed in this paper are fault dictionary approach, parameter identification approach, the fault verification approach and the approximation approach. A method is used which expands the harmonic balance technique from nonlinear simulation to non-linear ad joint sensitivity analysis [7]. The hierarchical approach is used to compute sensitivity analysis and calculates responses in any sub circuit in the hierarchy. Another multi frequency approach deals with the optimum selection of test points and frequencies [8]. The method presented is based on the large change sensitivity analysis and the deviation of the element values is taken from zero to infinity. Symbolic analysis is used in the calculation of the column rank of the sensitivity matrix [9]. Baoru et. al in [10] proposed a tolerance analog circuit hard and soft fault diagnosis method based on adoptive learning rate and additional momentum algorithm BP neural network.

\section{POLE ZERO ANALYSIS}

The Numerator and Denominator polynomials of the Transfer function are written as given in equation (1).

$$
\left.H(s)=\frac{N(S)}{D(S)}=K_{m} \frac{\left(s-z_{1}\right)\left(s-z_{2}\right) \ldots\left(s-z_{m}\right)}{\left(s-p_{1}\right)\left(s-p_{2}\right) \ldots\left(s-p_{n}\right)}\right)
$$

Where $K_{m}=a_{m} / b_{n}, s$ is the complex frequency and is equal to $s=\sigma+j \omega$. The roots $Z_{i}$ of the numerator polynomial $N(s)(N(s)=$ $0)$ are called Zeros of the transfer function and the roots of the denominator polynomial $D(s)(D(s)=0)$ are called Poles of the Transfer function, $m$ and $n$ are the numbers of the Zeros and Poles respectively. The coefficients of the numerator and denominator polynomials are real, therefore the Poles and Zeros must be either be real or appear in complex conjugate pairs. The Poles of the network depend only on the network structure and are independent of the input excitation. Whereas the Zeros of the network depend on the place to which the source is applied and also depend on the point where the output is measured.

The Poles and Zeros of the linear circuit together with the real constant $K_{m}$ or $K_{0}$ completely determine the transfer function as a function of the complex frequency $\mathrm{s}$. The transfer function is normally represented by the Pole and Zero diagram of the complex frequency $\mathrm{s}$, whose axis represents the real and imaginary parts of the complex variable $\mathrm{s}$.

\section{POLE SENSITIVITY ANALYSIS}

In [11] a method based on sensitivity investigation of the test characteristics in the frequency domain has been presented. The effect of parameter changes on performance specifications caused by the deviation of the manufacturing process can be determined by using the sensitivity analysis in [12].

To test analog circuits lot of information has to be provided to test engineers. For fault diagnosis of analog circuits, information like number of testable and non-testable components, ambiguity groups etc. are needed. The sensitivity analysis based on a first order approximation provides the variation of a circuit's response w.r.t parameter variations.

There are two forms of sensitivity- normalized differential sensitivity and normalized incremental sensitivity. The normalized differential sensitivity is given by the equation (2) and the incremental differential sensitivity is calculated using (3).

$$
S_{h_{i}}^{T_{j}}=\frac{h_{i}}{T_{j}} \frac{\partial T_{j}}{\partial h_{i}} .
$$




$$
\rho_{h_{i}}^{T_{j}}=\frac{h_{i}}{T_{i}} \frac{\Delta T_{j}}{\Delta h_{i}}
$$

In this paper Pole-Zero analysis as proposed in [11] has been used in the fault diagnosis of analog circuits which enables the time and frequency responses to be calculated easily. The calculation of Pole -Zero sensitivity analysis has wide range of applications:

- $\quad$ Provides fundamental information about the system stability

- Allows the designers to follow the direction and magnitude changes caused by the changes in element values

- Helps in extracting the behavioral model of the analog circuits

- Helps in calculation of transient and AC sensitivities

In this paper Pole-Sensitivity has been used in association with the Multi-Frequency approach to diagnose faults along with other known methods. This has increased the number of faults that are diagnosed successfully.

\section{ALGORITHM 1}

Step 1: The circuit is modeled using the transfer function in S- Domain.

\section{Step 2: The Poles and Zeros of the circuit are determined form the transfer function calculated in Step 1.}

Step 3: Using Adjoint method the Pole sensitivity with respect to the circuit elements is calculated.

\section{Step 4: Ambiguity groups are formed from the pole sensitivities.}

Step 5: If the ambiguity group from step 4, consists of more than one fault signature in a group, Multi frequency approach is used to isolate faults.

\section{Example 1: Band Pass Filter}

The Figure 1 shows a simple BP filter, which has been taken for analysis.

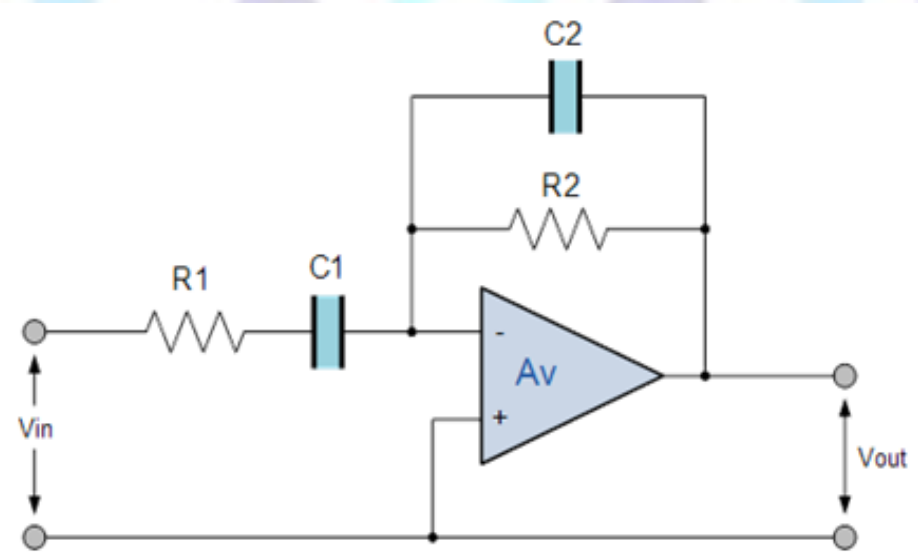

Figure 1: Band Pass Filter

In this filter $R_{1}=10 K \Omega, R_{2}=20 K \Omega, C_{1}$ and $C_{2}=11.2 \mathrm{nF}$. The transfer function of this circuit is given by:

$$
\mathrm{H}(\mathrm{s})=\frac{-R_{2} C_{1} S}{S^{2} R_{1} C_{1} R_{2} C_{2}+S\left(R_{1} C_{1}+R_{2} C_{2}\right)+1}
$$

The sensitivity of the poles under nominal case (without faults) is calculated using equation (2) and (3) and the results are tabulated in Table 1.

Table 1: Sensitivity of Poles -Nominal Case

\begin{tabular}{|c|c|c|}
\hline Component & $S_{h}^{P 1}$ & $S_{h}^{P 2}$ \\
\hline$R_{1}$ & -1.000 & -0.9612 \\
\hline$R_{2}$ & 0 & 0 \\
\hline$C_{1}$ & -1.000 & -0.9612 \\
\hline$C_{2}$ & 0 & 0 \\
\hline
\end{tabular}

Now the BP filter has been induced with eight different faults. The Table 2 gives the different types of faults that are assumed to be present in the circuit. 
Table 2: Fault Types

\begin{tabular}{|c|c|c|}
\hline S.No & Type of Fault & Name \\
\hline 1 & $\mathrm{R}_{1}+10 \%$ & $\mathrm{~F}_{1}$ \\
\hline 2 & $\mathrm{R}_{1}-10 \%$ & $\mathrm{~F}_{2}$ \\
\hline 3 & $\mathrm{R}_{2}+10 \%$ & $\mathrm{~F}_{3}$ \\
\hline 4 & $\mathrm{R}_{2}-10 \%$ & $\mathrm{~F}_{4}$ \\
\hline 5 & $\mathrm{C}_{1}+10 \%$ & $\mathrm{~F}_{5}$ \\
\hline 6 & $\mathrm{C}_{1}-10 \%$ & $\mathrm{~F}_{6}$ \\
\hline 7 & $\mathrm{C}_{2}+10 \%$ & $\mathrm{~F}_{7}$ \\
\hline 8 & $\mathrm{C}_{2}-10 \%$ & $\mathrm{~F}_{8}$ \\
\hline
\end{tabular}

The sensitivity of Poles is calculated for the circuit under fault condition and the results are shown in Table 3.

Table 3: Sensitivity of Poles -Faulty Case

\begin{tabular}{|c|c|c|}
\hline Faults & $S_{h}^{P 1}$ & $S_{h}^{P 2}$ \\
\hline$F_{1}$ & -0.9091 & 0 \\
\hline$F_{2}$ & -1.1111 & -0.8739 \\
\hline$F_{3}$ & 0 & -1.0680 \\
\hline$F_{4}$ & 0 & 0 \\
\hline$F_{5}$ & -0.9091 & -0.8739 \\
\hline$F_{6}$ & -1.1111 & -1.0680 \\
\hline$F_{7}$ & 0 & 0 \\
\hline
\end{tabular}

As seen from this Table 3 , the Faults are not isolated by this method. Also it has been found that the fault pairs $F_{1} F_{5}$, $F_{2} F_{6}$, $\mathrm{F}_{3} \mathrm{~F}_{7}$ and $\mathrm{F}_{4} \mathrm{~F}_{8}$ have the same fault signatures. These fault pairs form the ambiguity table shown in Table 4.

Table 4: Ambiguity Table

\begin{tabular}{|c|c|c|c|c|}
\hline Type & Gr 1 & Gr 2 & Gr 3 & Gr 4 \\
\hline Faults & $\mathrm{F}_{2} \mathrm{~F}_{6}$ & $\mathrm{~F}_{3} \mathrm{~F}_{7}$ & $\mathrm{~F}_{1} \mathrm{~F}_{5}$ & $\mathrm{~F}_{4} \mathrm{~F}_{8}$ \\
\hline
\end{tabular}

The Multi Frequency Algorithm proposed in [3] has been applied to the Pole Sensitivity analysis of Table 2 to diagnose faults. The voltage gain of the Circuit Under Test (CUT) is calculated at three different frequencies, cut-off frequency, one above the cut-off frequency and the other below the cut-off frequency. The gain of the CUT is normalized if necessary. The Gain vs Frequency is tabulated in Table 5 . The three frequencies are $\mathrm{f}_{1}=1 \mathrm{KHz}, \mathrm{f}_{2}=1.2 \mathrm{KHz}$ and $\mathrm{f}_{3}=800 \mathrm{~Hz}$.

Table 5: Gain vs Frequency

\begin{tabular}{|c|c|c|c|c|}
\hline \multirow{2}{*}{ S.No } & \multirow{2}{*}{ Fault } & \multicolumn{3}{|c|}{ Frequencies } \\
\cline { 3 - 5 } & & $\mathrm{f}_{1}$ & $\mathrm{f}_{2}$ & $\mathrm{f}_{3}$ \\
\hline 1 & $\boldsymbol{F}_{\mathbf{1}}$ & 678.58 & 762.73 & 587.1 \\
\hline 2 & $\boldsymbol{F}_{\mathbf{5}}$ & 715.73 & 807.38 & 587.14 \\
\hline 3 & $\boldsymbol{F}_{\mathbf{2}}$ & 704.08 & 798.89 & 621.27 \\
\hline 4 & $\boldsymbol{F}_{\mathbf{6}}$ & 660.63 & 748.26 & 568.03 \\
\hline 5 & $\boldsymbol{F}_{\mathbf{3}}$ & 695.3 & 777.7 & 591.52 \\
\hline 6 & $\boldsymbol{F}_{\mathbf{7}}$ & 695.3 & 784.38 & 591.52 \\
\hline 7 & $\boldsymbol{F}_{\mathbf{4}}$ & 671.17 & 823.97 & 608.59 \\
\hline 8 & $\boldsymbol{F}_{\mathbf{8}}$ & 671.16 & 755.01 & 608.59 \\
\hline
\end{tabular}


As seen the frequency $f_{1}$ could not isolate the faults $F_{3}, F_{7}, F_{4}$ and $F_{8}$ and the frequency $f_{3}$ could not isolate faults $F_{1}$, $F_{5}$, $F_{3}, F_{7}, F_{4}$ and $F_{8}$. The frequency $f_{2}$ has been able to isolate all the eight faults. Therefore the frequency $f_{2}=1.2 \mathrm{KHz}$ is the test frequency and has thus been able to isolate all the faults.

\section{RESULTS AND CONCLUSIONS:}

In this paper Pole Sensitivity analysis in association with Multi Frequency approach has been proposed. The algorithm proposed has been able to successfully isolate maximum number of faults. The algorithm has been applied on a Band Pass Filter and has been able to achieve $100 \%$ fault diagnosis.

\section{REFERENCES:}

[1] S.P. Venu Madhava Rao, N.Sarat Chandra Babu and K.Lal Kishore, "Fault diagnosis algorithm for analog electronic circuits based on Node-Frequency Approach", International Journal of Computer Science and Information Security, Vol. 8, No. 4, July 2010, pp. 291-298.

[2] S.P. Venu Madhava Rao, N.Sarat Chandra Babu and K.Lal Kishore, "Analog test zig for electronic circuit testing using FPGA's", Instrumentation Society of India, Vol.40, No.2, June 2010, pp. 122-123.

[3] N.Sarat Chandra Babu, V.C.Prasad, S.P. Venu Madhava Rao and K.Lal Kishore, "Multi Frequency approach to fault dictionary of linear analog fault diagnosis", Journal of Circuits, Systems and Computers, Vol.17, No. 5, October 2008, pp. 905-928.

[4] T.N. Trick and Y.Li, "A sensitivity based algorithm for fault isolation in analog circuits", Proceedings of International Symposium on Circuits and Systems, Vol.3, 1983, pp. 1098-1101.

[5] L.Rapisarda and R. DeCarlo, "Analog Multi frequency fault diagnosis", IEEE Transactions on Circuits and Systems, Vol. CAS-30, April 1983, pp. 223-234.

[6] John W Bandler and Aly E. Salama, "Fault Diagnosis of Analog circuits", Proceedings IEEE, Vol. 73, No. 8, August 1985, pp. 1279-1325.

[7] John .W. Bandler and Radslaw Biernacki, "A unified theory for frequency-domain simulation and sensitivity analysis of linear and nonlinear circuits", IEEE Transactions on Microwave Theory and Techniques, vol. 36, No. 12, December 1988.

[8] Tao Wei, Mike W.T. Wong, and Y.S. Lee, "Efficient multi frequency analysis of fault diagnosis in analog circuits based on large change sensitivity computation", IEEE, 1996, pp. 232-237.

[9] Tao Pi and C-J. Richard Shi, "Analog Testability analysis by Determinant Decision Diagrams based Symbolic Analysis", IEEE, pp.541-546.

[10] Baoru Han, Jingbing Li, Hengyu Wu, "Diagnosis method for Analog circuit Hard Fault and Soft fault", TELKOMNIKA, Vol. 11, No.9, 2013.

[11] Hasan Albustani, "Modelling Methods for Testability Analysis of Analog Integrated Circuits Based on Pole-Zero, AnalysisFakultät für IngenieurwissenschaftenIngenieurwissenschaften - Campus Duisburg » Abteilung Elektrotechnik und Informationstechnik.

[12] Elissaveta Dimitrova Gadjeva, Dimitar Yordanov Shikalanov and Anton Georgiev Atanasov, "Automated Observability Investigation of Analog Electronic Circuits using SPICE”, Annual Journal of Electronics, 2011.

\section{Author's Biography}

S.P. Venu Madhava Rao: He did his PhD from the prestigious JNTU, Hyderabad in the area of Analog VLSI Fault Diagnosis. His research areas include Nano Electronics, VLSI Analog Fault diagnosis, Machine learning etc. At present he is working as Professor and Head, Department of ECE in Sreenidhi Institute of Science and Technology, Hyderabad.

O.Manasa: She is pursuing her PG in VLSI \& ES and her research areas include VLSI Analog Fault Diagnosis. 